\title{
Diagnóstico precoce do câncer bucal com inteligência artificial: Uma revisão integrativa
}

\author{
Early diagnosis of oral cancer with artificial intelligence: An integrative review \\ Diagnóstico precoz del cáncer oral con inteligencia artificial: Revisión integrativa
}

Recebido: 25/02/2021 | Revisado: 04/03/2021 | Aceito: 03/05/2021 | Publicado: 17/05/2021

Glauciele Souza de Santana

ORCID: https://orcid.org/0000-0002-1912-405X

Universidade Federal de Pernambuco, Brasil

E-mail: glauciele.ssantana@gmail.com

Marina Rosa Barbosa

ORCID: https://orcid.org/0000-0002-6440-6013

Faculdade de Odontologia do Recife, Brasil

E-mail: marinab.odonto@gmail.com

Júlia Vanessa Bezerra Lima

ORCID: https://orcid.org/0000-0001-5393-1843

Faculdade de Odontologia de Pernambuco, Brasil

E-mail: julia.vanessa1912@hotmail

Luiza Fernanda Correia Molina Cabral

ORCID: https://orcid.org/0000-0003-2006-0353

Faculdade de Odontologia de Pernambuco, Brasil

E-mail: luizafernandaaaa@gmail.com

Anna Carolina Vidal Moura

ORCID: https://orcid.org/0000-0001-7115-1437

Faculdade de Odontologia de Pernambuco, Brasil

E-mail:caroolmoura123@hotmail.com

Thainara Vitória Lima Alves

ORCID: https://orcid.org/0000-0001-7617-4291

Faculdade de Odontologia de Pernambuco, Brasil E-mail:thainara.vlima25@gmail.com

Aryana Rocha do Nascimento

ORCID: https://orcid.org/0000-0003-0764-133X

Universidade Tiradentes de Pernambuco, Brasil E-mail: aryanaarocha@hotmail.com

Maria Eduarda Batista da Silva

ORCID: https://orcid.org/0000-0002-8915-9063

Universidade Federal de Pernambuco, Brasil E-mail: maria.ebatista2souubit.com.br

Ana Beatriz Leme de Andrade

ORCID: https://orcid.org/0000-0003-0357-8417 Faculdade de Odontologia de Pernambuco, Brasil

E-mail: anabeatrizleme@ hotmail.com

Jéssica da Silva Cunha

ORCID: https://orcid.org/0000-0003-1570-2964

Faculdade de Odontologia de Pernambuco, Brasil

E-mail:jc.odonto.2018@gmail.com

\begin{abstract}
Resumo
O câncer oral é uma neoplasia que acomete a região labial e a cavidade bucal, considerado o tipo mais comum de neoplasia no Brasil e no mundo, ocupando a sexta posição nos índices de mortalidade por câncer. Segundo o Instituto Nacional do Câncer (INCA) (2020), possui uma estimativa de cerca de 15.190 novos casos anuais de câncer bucal no Brasil. O objetivo desse estudo foi realizar uma revisão integrativa da literatura atual sobre o diagnóstico precoce do câncer oral com precisão, utilizando as ferramentas de inteligência artificial, destacando sua técnica de realização, indicações, limitações, vantagens e desvantagens. Uma revisão integrativa da literatura foi realizada, por meio de uma busca de artigos científicos na base de dados eletrônica PubMed, Scielo e Medline, utilizando os descritores: mouth neoplasms, diagnosis and artificial intelligence, indexados no período de 2016 a 2020, que tratavam de relato de caso, estudo in vitro e ex vivo. Após os critérios de elegibilidade, foram analisados 11 artigos integralmente publicados em língua inglesa. Os estudos mostraram que a técnica traz mais segurança para o diagnóstico precoce do câncer oral em pacientes assintomáticos ou sob suspeita, diminuindo as chances de evolução da neoplasia, contribuindo para melhores condutas de tratamento.
\end{abstract}


Palavras-chave: Neoplasias bucais; Diagnóstico; Inteligência artificial.

\begin{abstract}
Oral cancer is a neoplasm that affects the labial region and the oral cavity, considered the most common type of neoplasia in Brazil and in the world, occupying the sixth position in cancer mortality rates. According to the National Cancer Institute (INCA), 2020, it has an estimate of about 15,190 new cases of oral cancer in Brazil. The aim of this study was to carry out an integrative review of the current literature on the early diagnosis of oral cancer with precision using artificial intelligence tools, highlighting its technique, indications, limitations and disadvantages. An integrative literature review was carried out through a search for scientific articles in the electronic database PubMed, Scielo and Medline using the descriptors: oral neoplasms, diagnosis and artificial intelligence, indexed in the period from 2016 to 2020 that dealt with case reports , in vitro and ex vivo study. After the eligibility criteria, 11 articles fully published in English were allowed. The advanced studies that the technique brings more to the early diagnosis of oral cancer in asymptomatic patients who are safe or under suspicion, decreasing according to the chances of evolution of the neoplasia, contributing to the best treatment approaches.
\end{abstract}

Keywords: Mouth neoplasms; Diagnosis; Artificial intelligence.

\title{
Resumen
}

El cáncer oral es una neoplasia que afecta una región labial y una cavidad oral, considerada el tipo de neoplasia más común en Brasil y en el mundo, ocupando el sexto lugar con tasas de mortalidad por cáncer. Según el Instituto Nacional del Cáncer (INCA), 2020, tiene un estimado de 15,190 nuevos casos de cáncer oral anualmente en Brasil. El objetivo de este estudio fue realizar una revisión integradora de la literatura actual sobre el diagnóstico precoz del cáncer oral con precisión utilizando herramientas de inteligencia artificial, destacando su técnica de realización, indicaciones, limitaciones, ventajas y desventajas. Se realizó una revisión integradora de la literatura mediante la búsqueda de artículos científicos en la base de datos electrónica PubMed, Scielo y Medline utilizando los descriptores: neoplasias bucales, diagnóstico e inteligencia artificial, indexados en el período de 2016 a 2020 que trataban de casos clínicos, estudio in vitro y ex vivo. Después de los criterios de elegibilidad, se analizaron 11 artículos publicados íntegramente en inglés. Los estudios demostraron que una técnica aporta poca seguridad para el diagnóstico precoz del cáncer oral en pacientes asintomáticos o sospechosos, disminuyendo las posibilidades de progresión de la neoplasia, contribuyendo a mejores enfoques de tratamiento.

Palabras clave: Neoplasias bucales, Diagnóstico; Inteligencia artificial.

\section{Introdução}

O câncer oral é a sexta doença maligna mais comum no mundo, é uma grande preocupação no Sudeste Asiático, principalmente por causa dos hábitos orais prevalentes de mascar betel, fumar e consumir álcool (Kumar, et al., 2016). Estimase que seja o nono câncer mais comum em todo o mundo, e sua taxa de incidência vem apresentando tendência de aumento. Apesar dos avanços em tratamentos médicos e cirúrgicos, a maioria dos pacientes são diagnosticados em estágio avançado e têm um prognóstico ruim (Lan Liaoa, et al., 2018), tendo uma taxa média de sobrevivência de 5 anos. Essa estimativa aumentou nas últimas décadas, dependendo da localização e estágio na hora do diagnóstico (Marina Curra, et al., 2016).

O diagnóstico definitivo de câncer bucal é obtido por biópsia, seguida de exame histológico de lesões suspeitas. Esse método envolve o exame da cavidade bucal e o uso de um bisturi ou biópsia por punção e processamento histopatológico, seguido pela interpretação de um especialista (Shihan Liu, et al., 2017).

Dessa forma, o objetivo do presente estudo é realizar uma revisão integrativa da literatura para investigar outras formas de diagnósticos que são eficazes e que utilize da tecnologia como facilitadora do diagnóstico de câncer bucal, assim como relatar sua aplicabilidade, limitações e vantagens.

\section{Metodologia}

\subsection{Estratégia de Busca}

O presente estudo trata-se de uma revisão integrativa da literatura, realizada por meio de uma busca de artigos científicos na base de dados eletrônicos PubMed, Scielo e Medline, utilizando os descritores "mouth neoplasms, diagnosis, artificial intelligence". Ao executar a pesquisa a respeito das palavras chaves, a fim de compor a Tabela 1, colocou-se em exposição a quantidade final de artigos selecionados, esclarecendo assim, o trajeto até chegar ao produto final. 
Figura 1. Fluxograma de estratégia de busca nas bases de dados.

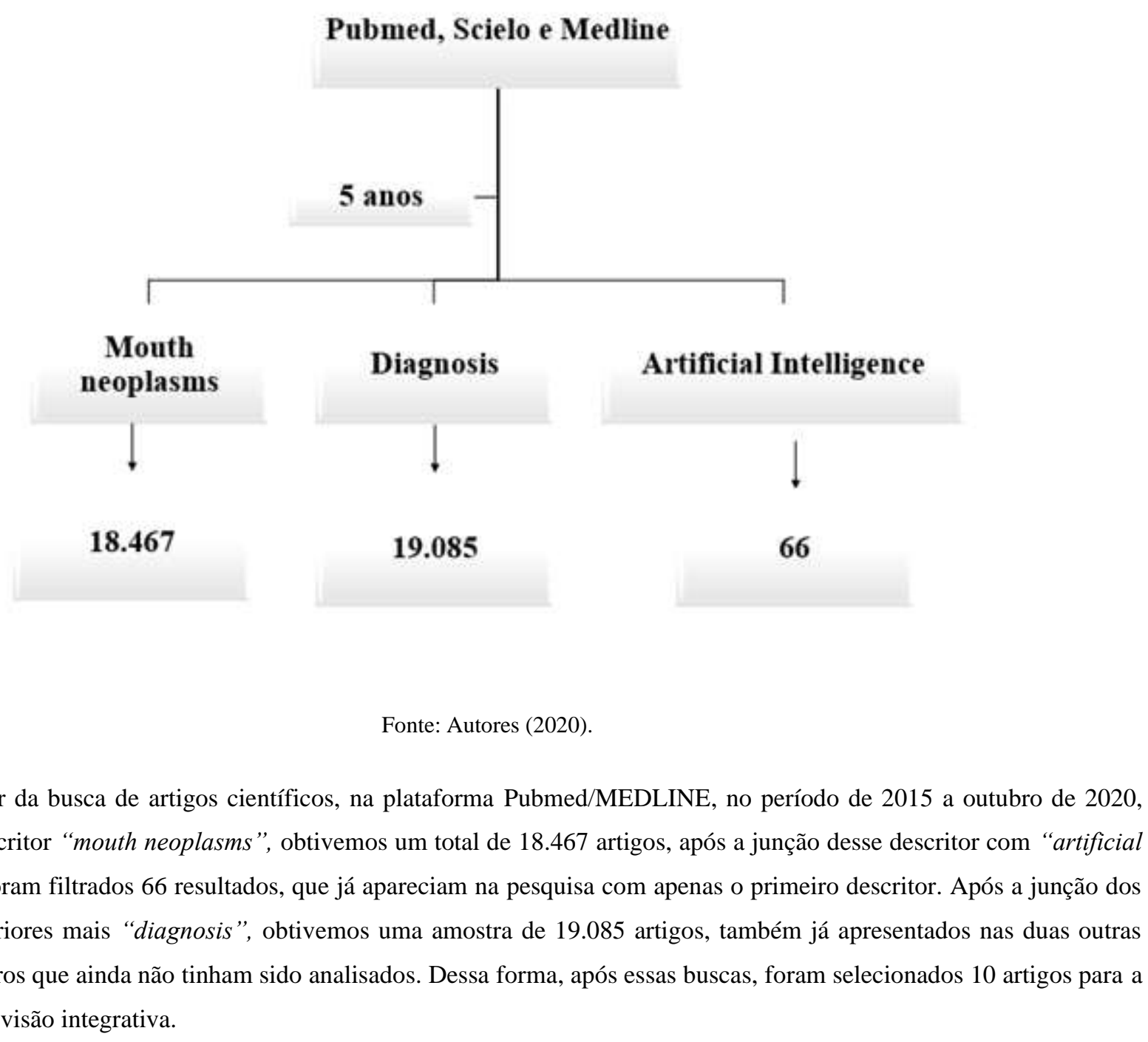

A partir da busca de artigos científicos, na plataforma Pubmed/MEDLINE, no período de 2015 a outubro de 2020 , utilizando o descritor "mouth neoplasms", obtivemos um total de 18.467 artigos, após a junção desse descritor com "artificial intelligence”, foram filtrados 66 resultados, que já apareciam na pesquisa com apenas o primeiro descritor. Após a junção dos descritores anteriores mais “diagnosis”, obtivemos uma amostra de 19.085 artigos, também já apresentados nas duas outras pesquisas, e outros que ainda não tinham sido analisados. Dessa forma, após essas buscas, foram selecionados 10 artigos para a elaboração da revisão integrativa.

\subsection{Critérios de elegibilidade dos artigos}

Para seleção das fontes, foram considerados como critérios de inclusão dos artigos originais disponibilizados na íntegra, publicados em língua inglesa e portuguesa, indexados no períodos de 2016 a agosto de 2020, artigos que visem o diagnóstico de câncer oral através de meios clínicos e laboratoriais, sem o auxílio de inteligência artificial e com o auxílio dessa tecnologia, estudos que relacionam a interação de inteligência artificial por meio de captação de imagens para o diagnóstico do câncer oral e estudos que utilizam inteligência artificial no diagnóstico precoce de neoplasia bucal. Não foram incluídos estudos de caso controle, pesquisa com uso de animais, artigos que relacionam o câncer oral com outras patologias, artigos que avaliam o uso de inteligência artificial em diagnóstico e terapia que não tem o câncer oral.

Segue a figura 2 que ilustra a estratégia de busca de acordo com os critérios citados. 
Figura 2. Fluxograma de estratégia de busca e seleção dos artigos.

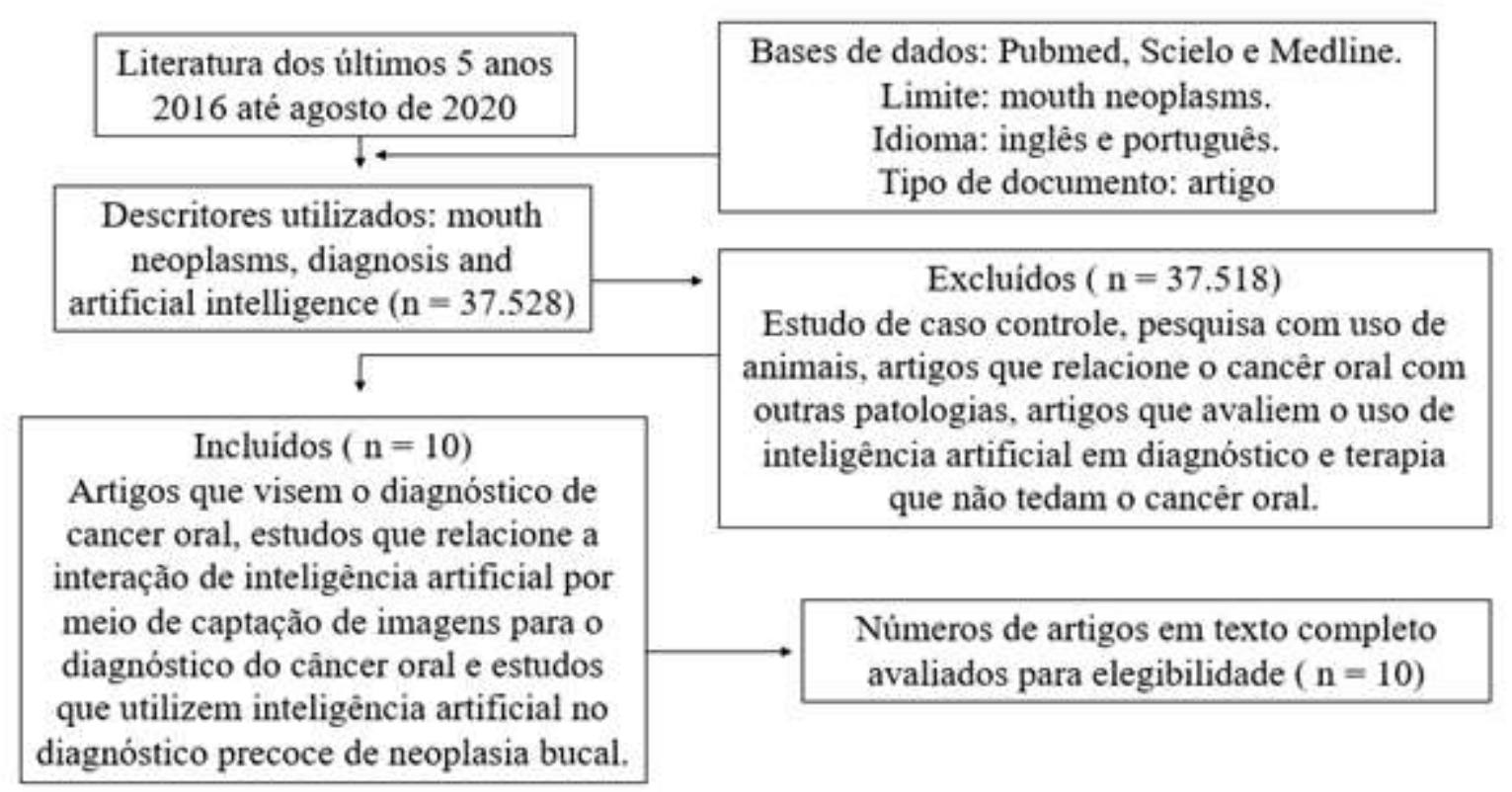

Fonte: Autores (2020)

Esta metodologia seguiu as recomendações PRISMA (Principais Itens para Relatar Revisões Sistemáticas e Metanálise). Os artigos que não se encaixavam nos critérios de inclusão, ou repetidos, foram excluídos. Posteriormente, dos artigos, restantes, foi realizada a leitura completa, chegando a uma amostra final de 10 artigos incluídos nesta revisão.

Não foram utilizadas teses, dissertações e monografias, visto que a realização de uma busca desses estudos é inviável logisticamente e a base de dados utilizada não tem esse tipo de publicação.

\section{Resultados e Discussão}

\subsection{Resultados}

Este estudo visou analisar a literatura sobre o câncer bucal, observando as vantagens do diagnóstico precoce do câncer utilizando da tecnologia artificial, desvantagens, período de demora do prognóstico e eficácia dessa nova possibilidade de diagnóstico, que possibilita maior rapidez na obtenção de um resultado. A presente revisão integrativa foi capaz de encontrar estudos recentes que trazem o surgimento da utilização da inteligência artificial, as formas de desenvolvê-la e os seus avanços, bem como a aplicação dessa nova técnica tanto para o diagnóstico como para medir potenciais de reincidência das lesões, como mostrado no Quadro 1. 
Quadro 1. Seleção dos artigos de acordo com o autor, tipo de estudo, objetivos, metodologia, resultados e conclusão.

\begin{tabular}{|c|c|c|c|c|}
\hline $\begin{array}{l}\text { Autor e } \\
\text { Estudo }\end{array}$ & Objetivo & Metodologia & Resultados & Conclusão \\
\hline $\begin{array}{l}\text { Alabi, et al., } \\
\quad(2019)\end{array}$ & $\begin{array}{l}\text { Avaliar in vivo uso de ANN } \\
\text { (redes neurais artificiais) no } \\
\text { prognóstico de OTSCC } \\
\text { (carcinoma de células } \\
\text { escamosas) precoce. }\end{array}$ & $\begin{array}{c}\text { Foram utilizados características clínico-patológicas de } 311 \\
\text { pacientes com cT1-2cNocM0 OTSCC tratado entre } 1979 \text { e } 2009 \text {, } \\
\text { nos Hospitais Universitários de Helsinque, Oulu,Turku, Tampere } \\
\text { e Kuopio na Filândia e e emo A.C. Camargo Cancer Center em } \\
\text { São Paulo, Brasil. }\end{array}$ & $\begin{array}{l}\text { Em termos de precisão da rede, a ANN rendeu uma } \\
\text { precisão geral de } 92,7 \% \text {. A precisão da RNA foi } \\
\text { maior do que a dada pelo modelo de regressão } \\
\text { logística que deu uma precisão de } 86,5 \% \text {. Além } \\
\text { disso, uma precisão geral de } 88,2 \% \text { foi obtida com a } \\
\text { Web ferramenta de prognóstico. }\end{array}$ & $\begin{array}{l}\text { O RNA parece oferecer um suporte único para a } \\
\text { tomada de decisão, prevendo recorrências e, } \\
\text { portanto, agregando valor para o gerenciamento de } \\
\text { OTSCC inicial. }\end{array}$ \\
\hline $\begin{array}{l}\text { Curra, et al., } \\
\quad(2016)\end{array}$ & $\begin{array}{l}\text { O objetivo deste estudo foi } \\
\text { avaliar a precisão da avaliação } \\
\text { clínica diagnóstico de lesões } \\
\text { labiais com base na } \\
\text { sensibilidade e especificidade. }\end{array}$ & $\begin{array}{l}\text { Foram usados dados em espécimes de biópsia labial registrados } \\
\text { entre } 1946 \text { e } 2011 \text { foram retirados do laboratório. Os dados } \\
\text { foram analisados usando o Statistical Package para as Ciências } \\
\text { Sociais. A precisão do diagnóstico foi calculada com base sobre } \\
\text { a sensibilidade, especificidade, razão de verossimilhança e valor } \\
\text { preditivo; a classificaçãa final dos diagnósticos (benigno, } \\
\text { potencialmente maligno e maligno) foi usado para esta avaliação. }\end{array}$ & $\begin{array}{l}\text { Nas primeiras duas décadas de vida, as lesões foram } \\
\text { mais prevalentes em mulheres; A maioria das lesões } \\
(\mathrm{n}=840,70,4 \%) \text { foram de indivíduos brancos. A } \\
\text { maioria }(\mathrm{n}=699,58,6 \%) \text { das lesões eram reativas, } \\
\text { seguidas por benignas neoplasias }(\mathrm{n}=238,19,9 \%), \\
\text { distúrbios do epitélio maturação }(\mathrm{n}=138,11,5 \%), \\
\text { neoplasias malignas }(\mathrm{n}=56,4,7 \%) \text {, outros }(\mathrm{n}=35, \\
2,9 \%) \text {, infeccioso lesões }(\mathrm{n}=20,1,7 \%) \text { e doenças } \\
\text { autoimunes }(\mathrm{n}=9,0,7 \%) \text {. Setenta e sete }(8,3 \%) \\
\text { casos foram diagnosticados como lesões malignas } \\
\text { ou PMD, com base em estudos histopatológicos } \\
\text { análise. A maioria dos pacientes diagnosticados } \\
\text { com lesões malignas estavam em sua quarta }(20,4 \%) \\
\text { e quinta }(32 \%) \text { décadas de vida. }\end{array}$ & $\begin{array}{l}\text { Os graus de sensibilidade específica variaram de } \\
34 \% \text { a } 77 \% \text { para lesões diferentes, e eram mais } \\
\text { elevados para autoimunes ( } 77 \%) \text { e lesões reativas } \\
\text { (72\%). Preditivo positivo e negativo os valores para } \\
\text { a identificação de PMD e lesões malignas foram } \\
81,9 \% \text { e } 98,9 \% \text {, respectivamente. O exame clínico } \\
\text { mostrou um alto grau de acurácia para detecção de } \\
\text { PMD e lesões malignas dos lábios, indicando boa } \\
\text { confiabilidade. }\end{array}$ \\
\hline $\begin{array}{l}\text { Kumar, et al., } \\
\quad(2016)\end{array}$ & $\begin{array}{l}\text { Descrever a visão geral dos } \\
\text { vários agentes etiológicos e } \\
\text { fatores de risco implicados no } \\
\text { desenvolvimento do câncer } \\
\text { oral. }\end{array}$ & $\begin{array}{l}\text { Foram utilizados para esse artigo de revisão de literatura: artigos } \\
\text { referente ao tema e descritores anexados. }\end{array}$ & $\begin{array}{l}\text { Os vários fatores de risco são implicado no } \\
\text { desenvolvimento de câncer oral, do qual o os mais } \\
\text { comuns e estabelecidos são o tabagismo, a } \\
\text { mastigação de betel quid e fatores como genéticos. } \\
\text { Acredita-se que a suscetibilidade desempenha o } \\
\text { papel causador. }\end{array}$ & $\begin{array}{l}\text { É de fundamental importância que o público e os } \\
\text { médicos tenham entendimento sobre os fatores de } \\
\text { risco para câncer bucal e é prudente para dentistas } \\
\text { olhar cuidadosamente para os primeiros sinais de } \\
\text { câncer bucal, enquanto exame de rotina da cavidade } \\
\text { oral, especialmente em pacientes com histórico de } \\
\text { fatores de risco conhecidos. }\end{array}$ \\
\hline $\begin{array}{c}\text { Skandarajah, et } \\
\text { al., (2017) }\end{array}$ & $\begin{array}{l}\text { Apresentar uma forma de } \\
\text { diagnóstico pontual, eficaz e } \\
\text { específico utilizando da } \\
\text { telemedicina. }\end{array}$ & $\begin{array}{l}\text { Microscópio baseado em tablet para diagnóstico remoto. O } \\
\text { dispositivo automatizado CellScope usa um iPad Mini para } \\
\text { coletar automaticamente uma série de pacientes imagens de } \\
\text { amostra, biópcia de escova para coletar o material. O aplicativo } \\
\text { possibilita acessar os dados do paciente e um servidor web foi } \\
\text { desenvolvido para armazenar imagens carregadas pelo } \\
\text { dispositivo CellScope e habilitar revisão remota dessas imagens } \\
\text { e dados associados por patologistas. Um menu pop-up de } \\
\text { características morfológicas permitiu a fácil seleção de todas as }\end{array}$ & $\begin{array}{l}\text { Os resultados indicaram que o instrumento coletou } \\
\text { com sucesso imagens de qualidade suficiente para } \\
\text { permitir diagnósticos remotos que mostram } \\
\text { concordância com as técnicas existentes. }\end{array}$ & $\begin{array}{l}\text { Este estudo demonstra o potencial do sistema de } \\
\text { microscopia móvel CellScope para permitir a } \\
\text { detecção precoce e prevenção do câncer bucal em } \\
\text { populações rurais como parte de um Sistema de } \\
\text { triagem baseado em telemedicina com avaliaçãa } \\
\text { remota de imagens. }\end{array}$ \\
\hline
\end{tabular}




\begin{tabular}{|c|c|c|c|c|}
\hline & & $\begin{array}{l}\text { características relevantes dentro da região selecionada. O estudo } \\
\text { foi desenhado para determinar a especificidade e sensibilidade de } \\
\text { uma preparação de citologia líquida de amostras adquiridas por } \\
\text { biópsia com escova emparelhadas com aquisição automática de } \\
\text { imagem. Pacientes que foram identificados a ter lesões } \\
\text { clinicamente suspeitas foram interrogados por citologia líquida e } \\
\text { transferência de dados realizado usando o microscópio móvel } \\
\text { automatizado. Os pacientes também foram submetidos a biópsia } \\
\text { com bisturi. O processamento histológico e o exame foram } \\
\text { realizados em MSMF de acordo com o padrão. }\end{array}$ & & \\
\hline $\begin{array}{l}\text { Sunny, et al., } \\
\quad(2019)\end{array}$ & $\begin{array}{c}\text { Detecção precoce do câncer } \\
\text { bucal requer um diagnóstico } \\
\text { minimamente invasivo e } \\
\text { específico do tecido } \\
\text { ferramenta que facilita a } \\
\text { triagem /vigilância. Dessa } \\
\text { forma, este trabalho tem como } \\
\text { objetivo o uso de um sistema } \\
\text { de telecitologia em } \\
\text { combinação com Rede Neural } \\
\text { Artificial (RNA) baseado } \\
\text { modelo de estratificação de } \\
\text { risco para detecção precoce de } \\
\text { potencialmente maligno oral } \\
\text { (OPML) / lesão maligna. }\end{array}$ & $\begin{array}{l}\text { Foram utilizado coleta de amostra e a preparação das lâminas, } \\
\text { imagens de citologia de alta resolução (2592 x 1936) de } \\
\text { profundidade de } 24 \text { bits com resolução óptica de 1,9 pixel / } \\
\text { micrômetro (resolução óptica 200X). } \\
\text { Foram capturados usando o CellScope, Esta plataforma capturou } \\
\text { os campos (100-125) em uma varredura raster padrão, as } \\
\text { imagens foram conectadas aos dados clínicos / demográficos dos } \\
\text { pacientes inseridos no aplicativo do iPad para o diagnóstico } \\
\text { remoto, os patologistas utilizaram um costume, interface do } \\
\text { portal da web. } \\
\text { Dois patologistas certificados conduziram uma revisão cega das } \\
\text { lâminas usando telecitologia e microscopia direta e documentou } \\
\text { as seguintes características: multi-nucleação, figuras mitóticas, } \\
\text { nucléolos proeminentes, relação nuclear citoplasmática alterada, } \\
\text { núcleo hipercromático, membrana nuclear irregular e quaisquer } \\
\text { características adicionais a seu critério. }\end{array}$ & $\begin{array}{l}\text { A eficácia geral (em detecção de OSCC e lesão de } \\
\text { HGD) de telecitologia com histologia como padrão } \\
\text { ouro foram } 62 \% \text { e } 69,5 \% \text { respectivamente. A } \\
\text { eficácia da citologia convencional usando } \\
\text { microscopia direta quando comparada à histologia } \\
\text { como o padrão ouro indicou que, conforme } \\
\text { observado no caso da telecitologia, as discrepâncias } \\
\text { estavam no diagnóstico de HGD. }\end{array}$ & $\begin{array}{c}\text { A plataforma de telecitologia avaliada neste estudo } \\
\text { é uma ferramenta eficaz para diagnóstico remoto } \\
\text { uma vez que poderia reter com sucesso recursos de } \\
\text { valor diagnóstico. Diagnóstico automatizado } \\
\text { baseado em ANN e a astratificação de risco } \\
\text { melhorou a sensibilidade na detecção de lesões de } \\
\text { HGD. Isto por sua vez,aumentou a precisão geral } \\
\text { do sistema em 30\% quando comparado ao método } \\
\text { manual. }\end{array}$ \\
\hline $\begin{array}{l}\text { Wong, et. al., } \\
\quad(2018)\end{array}$ & $\begin{array}{l}\text { Detecção precoce e tratamento } \\
\text { do câncer bucal. }\end{array}$ & $\begin{array}{l}\text { Avaliado sinais clínicos presentes nos pacientes através do } \\
\text { autoexame. }\end{array}$ & & \\
\hline $\begin{array}{l}\text { Lu, et al., } \\
\text { (2017) }\end{array}$ & $\begin{array}{l}\text { Investiga características de } \\
\text { imagem extraídas por } \\
\text { computador de forma nuclear } \\
\text { e textura em imagens } \\
\text { digitalizadas de seç̃oes de } \\
\text { tecido coradas com H\&E para } \\
\text { estratificação de risco de } \\
\text { pacientes com carcinoma de } \\
\text { células escamosas da cavidade } \\
\text { oral em comparação com } \\
\text { parâmetros clínicos e } \\
\text { patológicos padrão. }\end{array}$ & Análise de lâminas histológicas. & $\begin{array}{l}\text { O classificador utiliza imagens coradas com H\&E e } \\
\text { foi modelado em apenas pontos únicos de } \\
\text { microarray de tecido de } 2 \mathrm{~mm} \text { para cada tumor. Isso } \\
\text { sugere que amostras muito pequenas de tumor } \\
\text { podem ser suficientes para que esse teste seja } \\
\text { prognóstico, o que significa que ele deve funcionar } \\
\text { para tumores muito pequenos e também para } \\
\text { amostras de biópsia pequenas. }\end{array}$ & $\begin{array}{l}\text { Ele mostra capacidade prognóstica independente } \\
\text { para essas características morfológicas, fornecendo } \\
\text { fortes evidências de sua utilidade. Esses resultados } \\
\text { abrem caminho para estudos futuros para refinar } \\
\text { ainda mais o classificador e validá-lo de forma } \\
\text { independente em coortes de pacientes prospectivos. }\end{array}$ \\
\hline
\end{tabular}




\begin{tabular}{|c|c|c|c|c|}
\hline $\begin{array}{l}\text { Dhanuthai, et. } \\
\text { al., (2018) }\end{array}$ & $\begin{array}{l}\text { Este artigo analisa a literatura } \\
\text { atual e vários tópicos } \\
\text { polêmicos envolvendo o } \\
\text { diagnóstico e estratégias de } \\
\text { tratamento para pacientes com } \\
\text { cavidade oral / cancros da } \\
\text { orofaringe. }\end{array}$ & $\begin{array}{l}\text { Análise de prontuários das biópsias das instituições participantes } \\
\text { foram revisados para casos de câncer bucal diagnosticados no } \\
\text { período de } 2005 \text { a } 2014 \text {. }\end{array}$ & $\begin{array}{l}\text { Dos } 474.851 \text { casos acessados, } 6.151 \text { casos }(1,30 \%) \\
\text { foram diagnosticados na categoria de câncer bucal. } \\
\text { A média de idade dos pacientes foi } 58,37 \pm 15,77 \\
\text { anos. Um total de } 4.238 \text { casos }(68,90 \%) \text { foram } \\
\text { diagnosticados em homens, enquanto } 1.911 \text { casos } \\
\text { (31,07\%) foram diagnosticados em mulheres. }\end{array}$ & $\begin{array}{l}\text { O câncer bucal representa mortalidade e morbidade } \\
\text { significativas nos pacientes, principalmente quando } \\
\text { descoberto no final do curso da doença. }\end{array}$ \\
\hline $\begin{array}{l}\text { Bugter, et. al., } \\
\text { (2018) }\end{array}$ & $\begin{array}{l}\text { Investigar se a espectroscopia } \\
\text { de reflectância pode detectar } \\
\text { FC na mucosa bucal de } \\
\text { pacientes com câncer de } \\
\text { laringe.. }\end{array}$ & $\begin{array}{l}\text { Os dados desses dois parâmetros foram combinados para formar } \\
\text { um único 'biomarcador } \alpha \text { ', que discrimina de forma otimizada } \\
\text { esses dois grupos. }\end{array}$ & $\begin{array}{c}\text { O alfa foi menor no grupo com câncer de laringe } \\
(0,28) \text { do que no grupo controle }(0,30, p=0,007) \text {. } \\
\text { Alpha pode identificar pacientes oncológicos com } \\
\text { uma sensibilidade de } 78 \% \text { e uma especificidade de } \\
74 \% .\end{array}$ & $\begin{array}{l}\text { Esses resultados podem ser o primeiro passo para o } \\
\text { pré-rastreamento óptico do câncer de laringe. }\end{array}$ \\
\hline $\begin{array}{l}\text { Frare, et. al., } \\
\quad(2016)\end{array}$ & $\begin{array}{l}\text { Analisar o perfil clínico- } \\
\text { patológico de pacientes jovens } \\
\text { ( } \leq 40 \text { anos) com CEC oral e } \\
\text { correlacionar com um grupo } \\
\text { controle ( } \geq 50 \text { anos) por meio } \\
\text { de sistemas de graduação } \\
\text { histopatológica. }\end{array}$ & Testes Qui-quadrado e Exato de Fisher & $\begin{array}{l}\text { Não houve diferença na sobrevida global em } 5 \text { anos } \\
\text { entre os grupos estudados. Não houve diferença na } \\
\text { graduação histológica entre os grupos estudados de } \\
\text { acordo com os quatro sistemas utilizados. }\end{array}$ & $\begin{array}{l}\text { Este estudo mostrou que, apesar dos tumores } \\
\text { apresentarem grau histológico semelhante e mais } \\
\text { modalidades terapêuticas terem sido utilizadas no } \\
\text { grupo jovem, os tumores em pacientes jovens } \\
\text { apresentaram maior incidência de recorrência / } \\
\text { metástase, apresentando tendência a um } \\
\text { comportamento mais agressivo. }\end{array}$ \\
\hline $\begin{array}{l}\text { Carneiro-Neto, } \\
\text { et. al., (2017) }\end{array}$ & $\begin{array}{l}\text { Revisar sistematicamente a } \\
\text { literatura para identificar e } \\
\text { sugerir protocolos eficazes e } \\
\text { seguros para o manejo de } \\
\text { complicações orais em } \\
\text { pacientes oncológicos. }\end{array}$ & $\begin{array}{l}\text { A revisão sistemática foi elaborada pela PICO e PRISMA } \\
\text { incluindo critérios de elegibilidade e exclusão. }\end{array}$ & $\begin{array}{l}\text { Apenas } 06 \text { artigos foram incluídos na revisão } \\
\text { sistemática com exclusão de outros, por obter } \\
\text { pontuação } \geq 7 \text { com alta qualidade metodológica para } \\
\text { síntese dos manejos das complicações orais. Já os } 05 \\
\text { artigos foram associados a baixo risco de viés } \\
\text { compondo os protocolos sugestivos de manejos e a } \\
\text { metanálise em odds ratio }(0,916) \text { para cura e risco } \\
\text { relativo }(1,049) \text { para o desenvolvimento de mucosite } \\
\text { oral e dor. }\end{array}$ & $\begin{array}{l}\text { Os protocolos sugestivos de manejo da mucosite } \\
\text { oral e dor com MuGard - hidrogel mucoadesivo; } \\
\text { PerioAid Tratamiento® enxaguatório bucal anti- } \\
\text { séptico com clorexidina e cloreto de cetilpiridínio; } \\
\text { Episil@ plus benzydamine - gel bioadesivo } \\
\text { oromucoso; } 0,03 \% \text { de enxaguatório bucal Triclosan } \\
\text { Colgate Plax; e Diodo Laser de baixa intensidade } \\
\text { são seguros para pacientes oncológicos aplicados de } \\
\text { acordo com os parâmetros clínicos adotados. }\end{array}$ \\
\hline
\end{tabular}

Fonte: Autores (2020) 
Foram selecionados, dentre os critérios, 10 artigos científicos. Os resultados mostraram o emprego da oncologia e da telemedicina guiada como ferramenta para o diagnóstico de câncer bucal e lesões potencialmente malignas.

\subsection{Discussão}

Somos de acordo com a literatura, pois a maioria das doenças são fatores genéticos e epigenéticos. O desenvolvimento de carcinoma espinocelular oral, ou de cabeça e pescoço (HNSCC), e carcinoma das glândulas salivares menores é influenciado por ambos os fatores: tabaco, álcool, dieta e nutrição, vírus, radiação, etnia, predisposição familiar e genética, candidíase oral, imunossupressão, uso de enxaguatório bucal, sífilis, fatores dentários, riscos ocupacionais.

$\mathrm{O}$ atraso estimado no diagnóstico de câncer bucal a partir do momento em que o paciente procura atendimento médico é de três meses, a falta de experiência de especialistas nas configurações de atenção primária é um fator importante. As lesões cancerosas nos lábios são frequentemente precedidas por mudanças perceptíveis na mucosa oral ou labial, também conhecido como PMD; esse processo permite aos médicos detectar e tratar eficazmente as lesões nas fases iniciais da carcinogênese oral.

A avaliação microscópica é necessária para estabelecer o diagnóstico final e, assim, o tratamento adequado em todos os casos de PMD e lesões malignas. Entretanto, o aumento do número de casos de câncer e atrasos no diagnóstico levam a um mau prognóstico. Ferramentas para auxiliar o exame visual tradicional são necessárias no ambiente de cuidados primários, no qual a maioria dos pacientes apresentam lesões.

Algumas técnicas estão sendo aprimoradas e em análise para o auxílio do diagnóstico de lesões bucais de potencial maligno, como o estudo de Skandarajah, et al., (2017) e Sunny, et al., (2019), nos quais, a partir da biópsia de escova, adaptouse um dispositivo baseado em produtos eletrônicos móveis, com as vantagens de escala e conectividade, para facilitar a imagem e a transmissão de uma biópsia por escova simplificada e minimamente invasiva.

Um sistema de apresentação e upload de dados foi desenvolvido e implantado no ambiente clínico para permitir a revisão e anotação de imagens usando uma plataforma de telecitologia usando imagens RGB brutas para avaliar as características morfológicas. A leitura e a interpretação das imagens digitais capturadas pelo CellScope substituíram a avaliação pela visualização direta das lâminas em um microscópio convencional. Dessa forma, a análise dos resultados indica que a concordância entre patologistas no diagnóstico feita usando imagens automatizadas do CellScope foi "substancial", conforme classificado pela estatística o valor de 0,695. Isso indica que as imagens CellScope forneceram diagnósticos suficientes, informação de qualidade aos patologistas para formarem suas opiniões, para que o acordo entre eles não fosse baseado apenas nos casos analisados.

Estudos mostram outra forma de diagnóstico fazendo o uso de RNA, uma ferramenta da web para a previsão de recorrência em estágio inicial OTSCC. Uma rede neural parece ter potencial para oferecer uma melhor abordagem para análises de dados e reconhecimento de padrões dentro dos dados. Ele pode construir um modelo estatístico não linear para examinar sistemas biológicos. Não há necessidade de identificar marcadores prognósticos chave ou formar uma hipótese em análises usando RNA.

\section{Considerações Finais}

Por fim, podemos afirmar que a literatura converge quanto à eficácia, praticidade, segurança e aplicabilidade de diagnósticos com o uso da tecnologia. Porém observamos que há necessidade de se desenvolver materiais mais acessíveis e com mais praticidade para o uso cotidiano, como também, estudos retrospectivos e randomizados relacionando o sucesso em relação ao diagnóstico mais rápido e preciso a longo prazo. Além disso, o sucesso dessa técnica não depende do nível de experiência do profissional, devido ao conjunto de informação já pré-existente nos programas que auxiliam no diagnóstico. 
Research, Society and Development, v. 10, n. 5, e57310513319, 2021

(CC BY 4.0) | ISSN 2525-3409 | DOI: http://dx.doi.org/10.33448/rsd-v10i5.13319

\section{Referências}

Alabi, R. O., Elmusrati, M., Sawazaki-Calone, I., Kowalski, L. P., Haglund, C., Coletta, R. D., Mäkitie, A. A., Salo, T., Leivo, I. \& Almangush, A. (2019). Machine learning application for prediction of locoregional recurrences in early oral tongue cancer: a Web-based prognostic tool. Virchows Archiv, 475(4), 489-497.

Bugter, O., Hardillo, J. A., De Jong, R. J. B., Amelink, A., \& Robinson, D. J. (2018). Optical pre-screening for laryngeal cancer using reflectance spectroscopy of the buccal mucosa. Biomedical optics express, 9(10), 4665-4678.

Carneiro-Neto, J. N. (2017). Protocols for management of oral complications of chemotherapy and/or radiotherapy for oral cancer: Systematic review and meta-analysis current. Medicina oral, patologia oral y cirugia bucal, 22(1).

Curra, M., Salvadori, G., Junges R., Filho Sant'ana, M., Hugo, F. N., \& Martins, M. D. (2016). Accuracy of clinical diagnosis for the identification of potentially malignant disorders and malignant lip lesions. Brazilian oral research, 30(1).

Dhanuthai, K., Rojanawatsirivej, S., Thosaporn, W., Kintarak, S., Subarnbhesaj, A., Darling, M., Kryshtalskyj, E., Chiang, C. P., Shin, H. I., Choi, S. Y., Lee, S. S., \& Shakib, P. A. (2018). Oral cancer: A multicenter study. Medicina oral, patologia oral y cirugia bucal, 23(1).

Frare, J. C., Sawazaki-Calone, I., Ana-Lucia-Carrinho Ayroza-Rangel, A., Bueno, G., de Morais, C. F., Nagai, H. M., Kunz, R., \& Lopes, M. A. (2016). Histopathological grading systems analysis of oral squamous cell carcinomas of young patients. Medicina oral, patología oral y cirugía bucal, 21(3).

Kumar M., Nanavati R., Modi T. G., \& Dobariya C. (2016). Oral cancer: Etiology and risk factors: A review. Journal of cancer research and therapeutics, 12(2), 458 .

Lu, C., Lewis, J. S., Dupont, W. D., Plummer, W. D., Janowczyk, A., \& Madabhushi, A. (2017). An oral cavity squamous cell carcinoma quantitative histomorphometric-based image classifier of nuclear morphology can risk stratify patients for disease-specific survival. Modern Pathology, 30(12), 1655-1665.

Skandarajah, A., Sunny, S. P., Gurpur, P., Reber, C. D., D’Ambrosio, M. V., Raghavan, N., James, B. L., Ramanjinappa, R. D., Suresh, A., Kandasarma, U., Birur, P., Kumar, V. V., Galmeanu, H. C., Itu, A. M., Modiga-Arsu, M., Rausch, S., Sramek, M., Kollegal, M., Paladini, G., Kuriakose, M., Ladic, L., Koch, F. \& Fletcher, D. (2017). Mobile microscopy as a screening tool for oral cancer in India: A pilot study. PloS one, 12(11).

Sunny, S., Baby, A., James, B. L., Balaji, D., N. V. A., Rana, M. H., Gurpur, P., Skandarajah, A., D'Ambrosio, M., Ramanjinappa, R. D., Mohan, S. P., Raghavan, N., Kandasarma, U., Sangeetha, N., Hedne, N., Koch, F., Fletcher, D. A., Selvam, S., Kollegal, M., N. P. B., Ladic, L., Suresh, A., Pandya, H. J. \& Kuriakose, M. A. (2019). A smart tele-cytology point-of-care platform for oral cancer screening. PloS one, 14(11). 